\title{
Three-Body Dynamics and Self-Powering of an Electrodynamic Tether in a Plasmasphere
}

\author{
D. Curreli* and E. C. Lorenzini ${ }^{\dagger}$ \\ University of Padova, 35131 Padova, Italy \\ C. Bombardelli, ${ }^{\ddagger}$ M. Sanjurjo-Rivo, ${ }^{8}$ and J. Pelaez \\ Universidad Politécnica de Madrid, 28040 Madrid, Spain \\ D. Scheeres** \\ University of Colorado at Boulder, Boulder, Colorado 80309 \\ and \\ M. Lara ${ }^{\text {t† }}$ \\ Real Instituto y Observatorio de la Armada en San Fernando, 11110 Cadiz, Spain
}

\begin{abstract}
The dynamics of an electrodynamic tether in a three-body gravitational environment are investigated. In the classical two-body scenario the extraction of power is at the expense of orbital kinetic energy. As a result of power extraction, an electrodynamic tether satellite system loses altitude and deorbits. This concept has been proposed and well investigated in the past, for example for orbital debris mitigation and spent stages reentry. On the other hand, in the three-body scenario an electrodynamic tether can be placed in an equilibrium position fixed with respect to the two primary bodies without deorbiting, and at the same time generate power for onboard use. The appearance of new equilibrium positions in the perturbed three-body problem allow this to happen as the electrical power is extracted at the expenses of the plasma corotating with the primary body. Fundamental differences between the classical twobody dynamics and the new phenomena appearing in the circular restricted three-body problem perturbed by the electrodynamic force of the electrodynamic tether are shown in the paper. An interesting application of an electrodynamic tether placed in the Jupiter plasma torus is then considered, in which the electrodynamic tether generates useful electrical power of about $1 \mathrm{~kW}$ with a 20 -km-long electrodynamic tether from the environmental plasma without losing orbital energy.
\end{abstract}

\section{Nomenclature}

gravitational force

tether length

$=$ spacecraft total mass

$=$ electron mass

$=$ electron charge magnetic field vector

$=$ distance between the primaries

$=$ motional electric field

$=$ projection of $\mathbf{E}$ along the tether

$=$ electrodynamic force

specific electrodynamic force

= specific gravitational force

$=$ mass of the first primary (Jupiter)

$=$ mass of the second primary (Io)

$=$ local electron density of plasmasphere
$\mathbf{R}$

$R$
$R_{j}$
$\hat{\boldsymbol{u}}$
$\mathbf{v}_{\mathrm{pl}}$
$\mathbf{v}_{\mathrm{sc}}$
$\mathbf{v}_{\mathrm{rel}}$
$w$
$\theta$
$\mu=G M$
$\phi_{t}$
$\phi_{p}$
$\varphi$
$\boldsymbol{\Omega}=(0,0, \Omega)$
$\Omega=\sqrt{\frac{G\left(M_{1}+M_{2}\right)}{d^{3}}}$

$\omega$
$=$ position of the center of mass of the electrodynamic tether with respect to the center of the corotating frame $=$ magnitude of $\mathbf{R}$

$=$ Jupiter equatorial radius

$=$ tether unit vector

$=$ velocity of the plasma

= spacecraft orbital velocity

$=$ relative velocity between the spacecraft and the plasmasphere

$=$ tether width

$=$ angle between the tether unit vector $\hat{\boldsymbol{u}}$ and magnetic field $\mathbf{B}$

$=$ gravitational parameter

$=$ tether electric potential

$=$ plasma electric potential

$=$ angle between $\mathbf{B}$ and $\mathbf{v}_{\mathrm{rel}}$

$=$ angular velocity of revolution of the two primaries

$=$ Keplerian angular velocity of revolution of the two primaries

$=$ angular velocity of the body

\section{Subscripts}

$\begin{array}{ll}\mathrm{sc} & =\text { spacecraft } \\ 1 & =\text { first primary (Jupiter) } \\ 2 & =\text { second primary (Io) }\end{array}$

\section{Introduction}

HE three-body orbital dynamics of an electrodynamic tether (EDT) is investigated, together with power-extraction capabilities when the EDT is placed in the equilibrium positions of the perturbed circular restricted three-body problem. 
The orbital dynamics of EDTs have usually been investigated with a two-body description of the motion of the system's center of mass around the central body. The tether is modeled as a rigid dumbbell, with attitude uncorrelated to its orbital dynamics. Even with such a simple model, complex dynamical features and nontrivial stability properties appear, as already outlined by many authors (see $[1,2])$. The Lorentz force coming from the interaction of the cable with the plasmasphere can conveniently be used for propellantless propulsion [3], for example in orbit raising and lowering maneuvers. Several applications of such a concept have been considered in the past (see [4-7]). Significant flight and development activities have also been conducted on EDT systems (TSS-1, TSS-1R, PMG, and ProSEDS). Furthermore, EDTs have been demonstrated to be capable of extracting power from space plasmas, i.e., from the plasmasphere of a planet or the solar wind [8]. Relevant applications at Jupiter have been proposed [9-12]. For these reason an EDT can seriously be considered as a future efficient tool for power generation and maneuvering in all space applications where an appropriate plasmasphere (intended as plasma plus magnetic field) is present [13].

The dynamics of an EDT in a three-body gravitational environment has received a lesser attention than the two-body case. Pioneering studies of tethers in the three-body environment were done by Colombo [14] and, later, by Farquhar [15]. These studies investigated a cable-connected satellite for station-keeping around a collinear libration point. Subsequent works were done by Misra et al. [16] and Wong and Misra [17,18], and recently by Peláez and Scheeres $[19,20]$ and Sanjurjo-Rivo et al. [21]. A recent study lead by the Advanced Concept Team of the European Space Agency investigated the dynamics of tethered satellites at Lagrangian points $[22]^{+*}$. The lack of studies of EDTs in the three-body is justifiable when we consider that in the Earth-moon system the presence of a third body, the moon, can be treated just like a perturbation for lowEarth-orbit applications. This is not true when we deal with more general cases and with planetary systems like Jupiter.

Jupiter offers a unique environment for an EDT, due to the outstanding morphology of its magnetosphere and the fast rotation of the planet. All around the orbit of the Galilean moon Io, a region of increased plasma density called the plasma torus modifies and affects the Jupiter magnetosphere. The presence of the plasma torus is a consequence of the volcanic activity of the moon Io and its strong tidal heating. The motion of a spacecraft within the Torus is influenced by two main gravitational attractors, Jupiter and Io. Furthermore, all the Lagrangian points of the Jupiter-Io-spacecraft system are contained inside the torus. At first approximation, the motion of an EDT-spacecraft inside the torus can be described by the equations of the circular restricted three-body problem, with the addition of the electrodynamic force produced by the interaction of the EDT with the plasmasphere.

In this paper we show that a whole family of new dynamical features appears when we study the tether dynamics by using the dynamical paradigm of the circular restricted three-body problem. Relevant consequences on power production and spacecraft maneuvering can be inferred, like for example the possibility to extract power while maintaing a quasistable position with respect to the corotating frame. As an example, the case of Jupiter-Io EDT system is considered and analyzed here in detail.

The paper is organized as follows. The basic principles of an EDT operating in the generator mode are recalled, in order to show the relations describing the self-powering of a bare EDT. After that the three-body dynamical model has been derived, and the equilibrium positions obtained. A new family of equilibrium positions (extending the classic Lagrangian points) appears as a function of the electrodynamic force. Energy considerations lead to the evaluation of the electrical power that can be extracted from the environmental plasma. We have obtained a simplified model for the evaluation of the equilibrium positions, and we have verified the results with a higher fidelity numerical model. The model has then been applied to the planetary system composed by Jupiter-Io EDT, showing that kilowatts of useful power can be obtained by using tethers of moderate length, in condition of optimal matching of the load impedance.

\section{Power Generation Equations}

We consider a bare EDT [23] of length $L$ and rectangular section $w \times h$ (tape tether), mounted on a satellite with a load impedance $Z_{c}$. The motion of electric charges of the conductor with respect to the background magnetic field is responsible for a motional electric field (EF) $\mathbf{E}$, given by:

$$
\mathbf{E}=\left(\mathbf{v}_{\mathrm{sc}}-\mathbf{v}_{\mathrm{pl}}\right) \times \mathbf{B}
$$

where the plasma is assumed to be frozen to the magnetic field. The motional EF projected along the tether line, $E_{t}=\mathbf{E} \cdot \hat{\boldsymbol{u}}$, provides the potential that drives the electrical current flowing along the tether. Figure 1 shows a schematic model of the tether.

When ohmic losses are neglected, the electric circuit equation is:

$$
E_{t} L=E_{t} z^{*}+Z_{c} I
$$

where it was assumed no impedance of the plasma contactor at the cathode, $Z_{c}$ is the impedance of the load, and $z^{*}$ is the zero-bias position along the tether, measured from the anodic tip. Ohmic losses cannot be neglected in presence of a dense plasmasphere, but they are negligible for the less dense plasma of Jupiter and a low-resistance wire. For the plasma density of Jupiter, the tether radius is much less than the debye length and the electron Larmor radius and, consequently, electrons are collected according to the Langmuir's orbital motion limited (OML) model $[24,25]$. Under the OML assumptions, the collected current is a function of the tether-line abscissa $z$ [23]:

$$
\frac{\mathrm{d} I}{\mathrm{~d} z}=\frac{2 w}{\pi} q_{e} n_{e} \sqrt{2 \frac{q_{e}}{m_{e}} E_{t}\left(z^{*}-z\right)}
$$

After analytic integration of Eq. (3) the current profile $I(z)$ can be obtained with the condition $I(z=0)=0$, and the length-averaged value $I_{\text {avg }}=(1 / L) \int_{0}^{L} I(z) \mathrm{d} z$ is given by (see [1] for the derivation):

$$
I_{\text {avg }}=I_{0}\left(1-\frac{2}{5} \zeta\right) \zeta^{3 / 2}
$$

where

$$
I_{0}=\frac{4}{3} \frac{w}{\pi} q_{e} n_{e} L^{3 / 2} \sqrt{2 \frac{q_{e}}{m_{e}} E_{t}}
$$

and $\zeta=z^{*} / L$ is the nondimensional zero-bias position.

The power that can be extracted at the load is a function of $\zeta$ :

$$
P=Z_{C} I_{C}^{2} \approx Z_{C} I_{\text {avg }}^{2}=I_{0} E_{t} L(1-\zeta) \zeta^{3 / 2}
$$

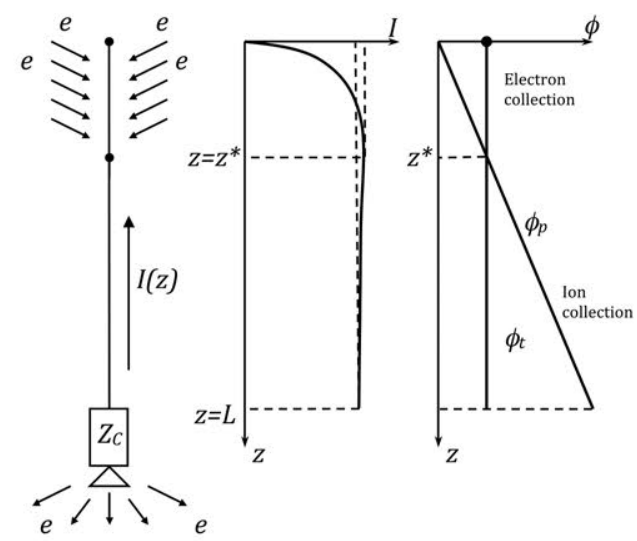

Fig. 1 Bare tether schematic (generator mode). The two graphs show the profiles of the current $I$, of the tether potential $\phi_{t}$ and of the plasma potential $\phi_{p}$ as a function of tether abscissa $z$. 
and it has a maximum at $\zeta_{\text {opt }}=3 / 5$. The optimal power condition can be obtained with an appropriate control of the load impedance. The optimal impedance $Z_{C}^{\mathrm{opt}}$ is proportional to the following set of parameters [1], which is variable along the trajectory as a function of $\sqrt{E_{t}} / N_{e}$

$$
Z_{C}^{\mathrm{opt}} \propto \frac{1}{N_{e} w} \sqrt{\frac{E_{t}}{L}}
$$

The Lorentz force due to the interaction of the current with the magnetic field is:

$$
\mathbf{F}_{\mathrm{el}}=\int_{0}^{L} I(z) \mathrm{d} \hat{\boldsymbol{u}} \times \mathbf{B}=I_{\mathrm{avg}} L B \hat{\boldsymbol{u}} \times \hat{\boldsymbol{b}}
$$

where $\hat{\boldsymbol{b}}$ is the unit vector of the magnetic field vector $\mathbf{B}$, and the current is parallel to the line element of the tether. The unit vector $\hat{\boldsymbol{u}}$ is assumed to have the same direction of the conventional electric current, pointing from the cathode to the anode. The tether current flow involves a particular caveat when dealing with rotating tethers, whereby cathode and anode reverse one another during each tether rotation. Substituting Eq. (4) in Eq. (8) yields:

$$
\mathbf{F}_{\mathrm{el}}=I_{0}\left(1-\frac{2}{5} \zeta\right) \zeta^{3 / 2} L B \hat{\boldsymbol{u}} \times \hat{\boldsymbol{b}}
$$

Equation (9) shows that the Lorentz force is proportional to:

$$
F_{\mathrm{el}} \propto n_{e} B E^{1 / 2} w L^{5 / 2} \propto n_{e} v_{\mathrm{rel}}^{1 / 2} B^{3 / 2} w L^{5 / 2}
$$

The scaling of the Lorentz force as a 2.5 power of the length enables the extraction of significant power levels from the plasmasphere using tethers of moderate lengths. In fact, the electrical energy extracted by the EDT during an infinitesimal time step is:

$$
\epsilon_{\text {load }}=\phi_{\text {load }} I_{\text {avg }} \mathrm{d} t
$$

and the associated electrical power is:

$$
P_{\mathrm{el}}=\mathbf{F}_{\mathrm{el}} \cdot \mathbf{v}_{\mathrm{rel}}=I_{0}\left(1-\frac{2}{5} \zeta\right) \zeta^{3 / 2} L B(\hat{\boldsymbol{u}} \times \hat{\boldsymbol{b}}) \cdot \mathbf{v}_{\mathrm{rel}}
$$

The maximum power can be obtained when the tether line $\hat{\boldsymbol{u}}$ is both perpendicular to the magnetic field and to the relative velocity vector; in this situation we have $(\hat{\boldsymbol{u}} \times \hat{\boldsymbol{b}}) \cdot \hat{\boldsymbol{v}}_{\text {rel }}=1$ and the power is:

$$
P_{\mathrm{el}}=\frac{4}{3} \frac{w}{\pi} q_{e} n_{e} v_{\mathrm{rel}} B L^{5 / 2} \sqrt{2 \frac{q_{e}}{m_{e}}} \sqrt{E_{t}}\left(1-\frac{2}{5} \zeta\right) \zeta^{3 / 2}
$$

Like the electrodynamic force, the electrical power $P_{\mathrm{el}}$ scales as $L^{5 / 2}$.

From a thermodynamic point of view, the EDT can be seen as a machine capable to convert directly the kinetic energy of its motion into electrical energy, by virtue of the induction law. The environmental plasma allows the closure of the electrical circuit and the possibility to have a current flowing through the tether and possibly a load. The kinetic energy associated with the relative motion is:

$$
\varepsilon_{\mathrm{kin}}^{\mathrm{rel}}=\frac{1}{2} m_{\mathrm{sc}} v_{\mathrm{rel}}^{2}=\frac{1}{2} m_{\mathrm{sc}}\left(\frac{E}{B \sin \varphi}\right)^{2}
$$

where $E=v_{\text {rel }} B \sin \varphi$, and $\varphi$ is the angle between $\mathbf{v}_{\text {rel }}$ and $\mathbf{B}$. The efficiency of power production of the EDT can be expressed as the ratio between the electrical power at the load and the total available power:

$$
\eta=\frac{P_{\mathrm{el}}}{\dot{\varepsilon}_{\mathrm{kin}}^{\text {rel }}}
$$

In the following discussion we will assume the load impedance being actively controlled (for example by using variable resistance), in order to track the condition for optimal matching.

\section{EDT Three-Body Motion}

The tethered satellite is assumed to have a rectilinear rigid shape of length $L$ with total mass $m$. The center of mass of the satellite is the point $C$ and the unit vector defining its orientation is $\hat{\boldsymbol{u}}$ (Fig. 2). The gravitational potential acting over an element mass $d m$ of the tether placed at a distance $R_{d m}$ from an attracting center is given by:

$$
V_{\mathrm{gr}}=-\int_{m} \frac{\mu}{R_{d m}} d m
$$

The ratio $1 / R_{d m}$ can conveniently be rewritten using Legendre polynomials:

$$
\frac{1}{R_{d m}}=\frac{1}{R \sqrt{1+2 \lambda \cos \gamma+\lambda^{2}}}=\frac{1}{R} \sum_{n=0}^{\infty}(-1)^{n} \lambda^{n} P_{n}[\cos \gamma]
$$

Substituting Eq. (17) into Eq. (16) the gravitational potential becomes:

$$
V_{\mathrm{gr}}=-\frac{m \mu}{R}\left(1+\sum_{n=2}^{\infty}(-1)^{n}\left(\frac{L}{R}\right)^{n} a_{n} P_{n}[\cos \gamma]\right)
$$

Equation (18) shows that the potential is given by the sum of a zeroth-order term $-m \mu / R$ corresponding to the potential of a point mass and a power series of the ratio $L / R$, where $L$ is the tether length and $R$ the distance of center of mass $C$ of the tether system from the attracting primary. The ratio $L / R$ is usually small and the corresponding terms negligible, but this condition does not hold for an extremely long tether or for a tether orbiting very close to one of the primaries.

The resultant of gravitational forces can similarly be derived:

$$
\begin{aligned}
\mathbf{F}_{\mathrm{gr}} & =-\frac{m \mu}{R^{3}} \mathbf{R}\left(1+\sum_{n=2}^{\infty}(-1)^{n}\left(\frac{L}{R}\right)^{n} a_{n}\left(S_{n}[\cos \gamma] \hat{\boldsymbol{R}}\right.\right. \\
& \left.\left.-S_{n-1}[\cos \gamma] \hat{\boldsymbol{u}}\right)\right)
\end{aligned}
$$

where the $S_{n}[\cos \gamma]$ are the polynomials of the series:

$$
\begin{gathered}
S_{1}[x]=3 x \\
S_{2}[x]=\frac{3}{2}\left(5 x^{2}-1\right) \\
S_{3}[x]=\frac{5}{2}\left(7 x^{3}-3 x\right) \\
S_{4}[x]=\frac{15}{8}\left(21 x^{4}-14 x^{2}+1\right)
\end{gathered}
$$

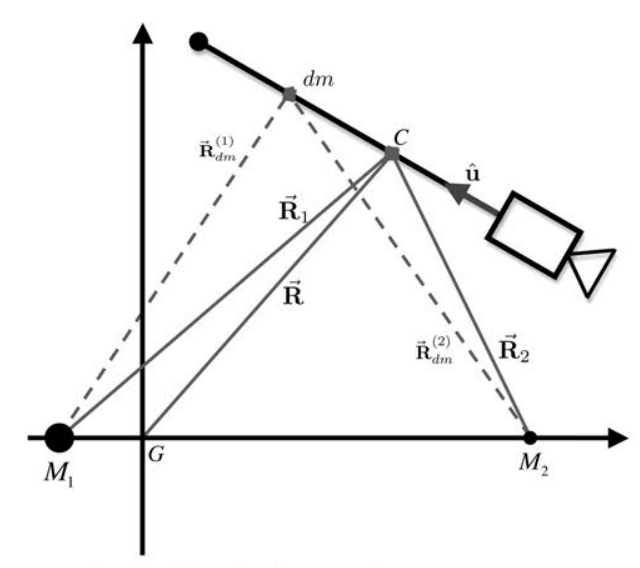

Fig. 2 Gravitational actions on the tether. 
In the limiting case when the ratio between tether length $L$ and distance $R$ from the gravitational attractor is small, the gravitational force coincides with the point-mass Newton law:

$$
L / R \rightarrow 0: \mathbf{F}_{\mathrm{gr}}=-\frac{m \mu}{R^{3}} \mathbf{R}
$$

When placed in a three-body environment the gravitational attractions of the two primaries $M_{1}$ and $M_{2}$ must be summed together. Here we will assume $M_{1}>M_{2}$, and we will indicate with the subscripts 1 and 2 the relative distances between the tether center of mass from $M_{1}$ and $M_{2}$, respectively. The motion of the center of mass, expressed in the synodic frame (the frame corotating with the primaries), is expressed by the classical circular restricted three-body problem (CRTBP) equations plus the perturbation due to the EDT electrodynamic force:

$$
\ddot{\mathbf{R}}+2 \boldsymbol{\Omega} \times \dot{\mathbf{R}}+\boldsymbol{\Omega} \times(\boldsymbol{\Omega} \times \mathbf{R})=-\frac{\mu_{1}}{R_{1}^{3}} \mathbf{R}-\frac{\mu_{2}}{R_{2}^{3}} \mathbf{R}_{2}+\mathbf{f}_{\mathrm{el}}
$$

with the assumption of a vanishing $L / R$ ratio. When the tether length is not negligible, the full gravitational force of Eq. (19) must be considered. The nondimensional form of Eq. (25) is:

$$
\begin{gathered}
\ddot{\xi}-2 \dot{\eta}=\xi-\frac{v_{1}}{\rho_{1}^{3}}\left(\xi+v_{2}\right)-\frac{v_{2}}{\rho_{2}^{3}}\left(\xi-v_{1}\right)+\frac{F_{\mathrm{el}, x}}{m d \Omega^{2}} \\
\ddot{\eta}+2 \dot{\xi}=\eta-\frac{v_{1}}{\rho_{1}^{3}} \eta-\frac{v_{2}}{\rho_{2}^{3}} \eta+\frac{F_{\mathrm{el}, y}}{m d \Omega^{2}} \\
\ddot{\zeta}=-\frac{v_{1}}{\rho_{1}^{3}} \zeta-\frac{v_{2}}{\rho_{2}^{3}} \zeta+\frac{F_{\mathrm{el}, z}}{m d \Omega^{2}}
\end{gathered}
$$

where $(\xi, \eta, \zeta)$ are the nondimensional coordinates of the orbital position. Length, mass, and time have been, respectively, nondimensionalized with the distance between primaries $d$, the total mass of the planetary system $M_{1}+M_{2}$, and the inverse of the mean angular velocity of the primaries $1 / \Omega$. Figure 3 shows the geometry of the synodic frame with the nondimensional coordinates $(\xi, \eta)$ centered in the center of mass of the planetary system (with $\left.M_{1}>M_{2}\right)$.

The nondimensional mass parameters of the two primaries are:

$$
\begin{gathered}
v_{1}=1-v_{2}=\frac{M_{1}}{M_{1}+M_{2}} \\
v_{2}=\frac{M_{2}}{M_{1}+M_{2}}
\end{gathered}
$$

and the nondimensional distances of the center of mass of the spacecraft from the two primaries are:

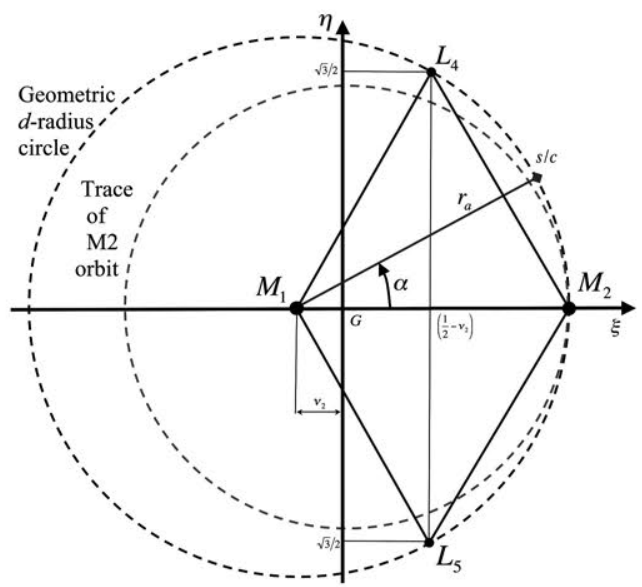

Fig. 3 Geometry of synodic plane with nondimensional quantities.

$$
\begin{aligned}
& \rho_{1}=\sqrt{\left(\xi+v_{2}\right)^{2}+\eta^{2}+\zeta^{2}} \\
& \rho_{2}=\sqrt{\left(\xi-v_{1}\right)^{2}+\eta^{2}+\zeta^{2}}
\end{aligned}
$$

From Eq. (31) and (32) together with Eqs. (26-28), it can be shown that in the unperturbed case the equilibrium points must lie in the intersections of two circumferences with their centers in $\left(-v_{2}, 0\right)$ and $\left(v_{1}, 0\right)$ and radii equal to $\rho_{1}$ and $\rho_{2}$ respectively.

The system of Eqs. (26-28) describes the dynamics of an active electrodynamic tether in a three-body case, which corresponds to a perturbation of the classical three-body problem, with a perturbing force due to the specific Lorentz force $\mathbf{f}_{\mathrm{el}}$.

When $\mathbf{f}_{\mathrm{el}}=0$, the system falls back to the case of the classical circular restricted three-body problem, with equilibrium positions consisting of the well-known five Lagrangian points. Small oscillations around Lagrangian points are expressed by the usual linear-variational solution of Szebehely [26]:

$$
\begin{gathered}
{\left[\begin{array}{l}
\xi \\
\eta
\end{array}\right]=\left[\begin{array}{c}
C_{1} \\
\bar{C}_{1}
\end{array}\right] \cos \left(s_{1} t\right)+\left[\begin{array}{c}
S_{1} \\
\bar{S}_{1}
\end{array}\right] \sin \left(s_{1} t\right)} \\
+\left[\begin{array}{c}
C_{2} \\
\bar{C}_{2}
\end{array}\right] \cos \left(s_{2} t\right)+\left[\begin{array}{c}
S_{2} \\
\overline{S_{2}}
\end{array}\right] \sin \left(s_{2} t\right)
\end{gathered}
$$

Eigenfrequencies at triangular points have the closed form

$$
s_{1,2}=\operatorname{Im}\left[\sqrt{(1 / 2)\left(-1 \pm \sqrt{1-27 v_{2}\left(1-v_{2}\right)}\right)}\right] \cdot \Omega[\mathrm{rad} / \mathrm{s}]
$$

expressing a motion that is a combination of short-period terms, associated with the eigenfrequency $s_{1}$ and long-period terms, associated with $s_{2}$. The two characteristic eigenfrequencies are a function of the planetary system parameter $v_{2}$.

The system of Eqs. (26-28) has no closed analytical solution, and a numerical analysis is required to solve for the trajectories. However, key features of this dynamical system can be derived with a qualitative dynamics analysis. In the following section equilibrium positions of the perturbed CRTBP are obtained, and the EDT equilibrium is analyzed.

\section{Equilibrium Positions}

When the EDT orbits in a three-body environment with a plasmasphere, the Lorentz force [Eq. (9)] perturbs the natural threebody motion and equilibrium positions different than Lagrangian points appear. When placed in these positions, the satellite can stay in equilibrium with respect to the synodic frame, because of the nonzero electrodynamic force acting on the tether.

The equilibrium positions in the perturbed case are the singular points (null acceleration and velocity) of the dynamical system described by Eqs. (26-28). The singular points are the solutions of the following equations:

$$
\begin{gathered}
\frac{v_{1}}{\rho_{1}^{3}}\left(\xi+v_{2}\right)+\frac{v_{2}}{\rho_{2}^{3}}\left(\xi-v_{1}\right)=\xi+f_{\xi} \\
\frac{v_{1}}{\rho_{1}^{3}} \eta+\frac{v_{2}}{\rho_{2}^{3}} \eta=\eta+f_{\eta} \\
\frac{v_{1}}{\rho_{1}^{3}} \zeta+\frac{v_{2}}{\rho_{2}^{3}} \zeta=f_{\zeta}
\end{gathered}
$$

where $f_{\xi}, f_{\eta}$ and $f_{\zeta}$ are the three nondimensional components of the perturbing force,

$$
\left(f_{\xi}, f_{\eta}, f_{\zeta}\right)=\left(F_{\mathrm{el}, x} / m d \Omega^{2}, F_{\mathrm{el}, y} / m d \Omega^{2}, F_{\mathrm{el}, z} / m d \Omega^{2}\right)
$$

with components expressed in the synodic frame. From the system of Eqs (35-37) the locations of the equilibrium positions $\left(\xi_{0}, \eta_{0}, \zeta_{0}\right)$ can be readily found, as a function of the perturbing electrodynamic 
force. Equation (37) shows that the equilibrium positions outside the orbital plane of the two primaries are theoretically possible:

$$
\zeta_{0}=\frac{f_{\zeta}}{v_{1} / \rho_{1}^{3}+v_{1} / \rho_{1}^{3}}
$$

When the out-of-plane force vanishes $f_{\zeta}=0$, the equilibrium positions lie on the orbital plane $\zeta_{0}=0$. In our simplified model of the electrodynamic force for the EDT we have assumed $f_{\zeta} \approx 0$; thus, no equilibrium positions exists in the out-of-plane region.

Making the substitution $\tau=1 / \rho_{1}^{3}$ and $\chi=1 / \rho_{2}^{3}$ the twodimensional system of Eqs. (35) and (36) yields:

$$
\left[\begin{array}{cc}
v_{1}\left(\xi+v_{2}\right) & v_{2}\left(\xi-v_{1}\right) \\
v_{1} \eta & v_{2} \eta
\end{array}\right]\left[\begin{array}{l}
\tau \\
\chi
\end{array}\right]=\left[\begin{array}{l}
\xi+f_{\xi} \\
\eta+f_{\eta}
\end{array}\right]
$$

The determinant of the matrix on the left-hand side of Eq. (40) is $v_{1} v_{2} \eta$, and for $\eta \neq 0$ the matrix can be inverted to obtain $(\tau, \chi)$. This corresponds to searching equilibrium positions outside the axis $\eta=0$ that joins the two primaries. Solving Eq. (40), the two distances $\rho_{1}, \rho_{2}$ of the equilibrium point from the two primaries are found as:

$$
\begin{aligned}
& \rho_{1}=\sqrt[3]{\frac{v_{1} \eta}{v_{1} \eta+f_{\xi} \eta+f_{\eta}\left(v_{1}-\xi\right)}} \\
& \rho_{2}=\sqrt[3]{\frac{v_{2} \eta}{v_{2} \eta-f_{\xi} \eta+f_{\eta}\left(v_{2}+\xi\right)}}
\end{aligned}
$$

For $\left(f_{\xi}, f_{\eta}\right)=(0,0)$ the external force vanishes, the distances from the primaries become unitary $\rho_{1}=\rho_{2}=1$, and the equilibrium positions are the classical triangular Lagrangian points: $\left(\xi_{0}, \eta_{0}\right)=$ $\left(1 / 2-v_{2}, \pm \sqrt{3} / 2\right)$.

For $\left(f_{\xi}, f_{\eta}\right)=\left(f_{\xi}, 0\right)$ the force acts purely in a direction parallel to the synodic $\xi$ axis. In this case, Eqs. (41) and (42) show that the dependence of $\rho_{1}, \rho_{2}$ on the $\xi$ coordinate disappears and an analytical solution of the equilibrium points $\left(\xi_{0}, \eta_{0}\right)$ can be readily obtained:

$$
\xi_{0}=\frac{1}{2}-v_{2}+\frac{1}{2}\left(\frac{1-v_{2}}{1-v_{2}+f_{\xi}}\right)^{2 / 3}-\frac{1}{2}\left(\frac{v_{2}}{v_{2}-f_{\xi}}\right)^{2 / 3}
$$

$$
\begin{aligned}
& \eta_{0}= \\
& \pm \sqrt{\left(\frac{1-v_{2}}{1-v_{2}+f_{\xi}}\right)^{2 / 3}-\left[\frac{1}{2}+\frac{1}{2}\left(\frac{1-v_{2}}{1-v_{2}+f_{\xi}}\right)^{2 / 3}-\frac{1}{2}\left(\frac{v_{2}}{v_{2}-f_{\xi}}\right)^{2 / 3}\right]^{2}}
\end{aligned}
$$

For $\left(f_{\xi}, f_{\eta}\right) \neq(0,0)$ the perturbing components are both different from zero; no closed form exist, and the equilibrium position must be derived by means of a numerical method.

Interesting properties of the equilibrium locations can be inferred when we shift to a polar coordinate system $\left(r_{a}, \alpha\right)$, by making the substitutions: $\xi=r_{a} \cos \alpha-v_{2}$ and $\eta=r_{a} \sin \alpha$, with $r_{a}$ the nondimensional radial distance from the first primary $M_{1}$ (see Fig. 3 ). In this new set of coordinates the distances from the primaries take the form:

$$
\begin{gathered}
\rho_{1}=r_{a} \\
\rho_{2}=\sqrt{r_{a}^{2}+1-2 r_{a} \cos \alpha}
\end{gathered}
$$

On the circle centered in $M_{1}$ and joining the triangular points with the second primary the radius $r_{a}$ is unitary, $r_{a}=1$, and the components of the force necessary for maintaining the equilibrium positions take the simple expression:

$$
\left[\begin{array}{l}
f_{\xi} \\
f_{\eta}
\end{array}\right]=v_{2}\left(\frac{1}{\rho_{2}^{3}}-1\right)\left[\begin{array}{c}
\cos \alpha-1 \\
\sin \alpha
\end{array}\right]
$$

where $\rho_{1}=1$ and $\rho_{2}=\sqrt{2-2 \cos \alpha}$. Equation (47) is the nondimensional force that the tether (or any other thrust-device) must supply to remain stationary with respect to the synodic reference frame at a given angular position $\alpha$ in the circle joining the triangular points with the second primary. In dimensional units the magnitude of the force is:

$$
F=m d \Omega^{2} \sqrt{f_{\xi}^{2}+f_{\eta}^{2}}=m d \Omega^{2} v_{2} \rho_{2}\left|\frac{1}{\rho_{2}^{3}}-1\right|
$$

Equation (48) shows that no external force is required to maintain an object at $|\alpha|=60 \mathrm{deg}$, that is at a triangular Lagrangian point, as expected. Figure 4 shows the two components $f_{\xi}, f_{\eta}$ of the electrodynamic force from Eq. (47) of the nondimensional force vs the angle $\alpha$, for all admissible values of the mass parameter $0<v_{2}<1 / 2$. Note that a null external force is required at the Lagrangian triangular points $\left(\alpha=60^{\circ}, \alpha=300^{\circ}\right)$. Singular conditions occur at $\alpha=0^{\circ}=360^{\circ}$, where the angular position of the second primary is reached by the spacecraft.

Figure 5 shows the nondimensional force as a vector field superimposed to the synodic plane of the Jupiter-Io system $\left(v_{2}=\right.$ $\left.4.7 \times 10^{-5}\right)$. In the figure Jupiter is placed at $\left(-v_{2}, 0\right)$, Io at $\left(v_{1}, 0\right)$, and the five Lagrangian points are marked with a cross. The circle centered in Jupiter and joining Io with the triangular points is marked

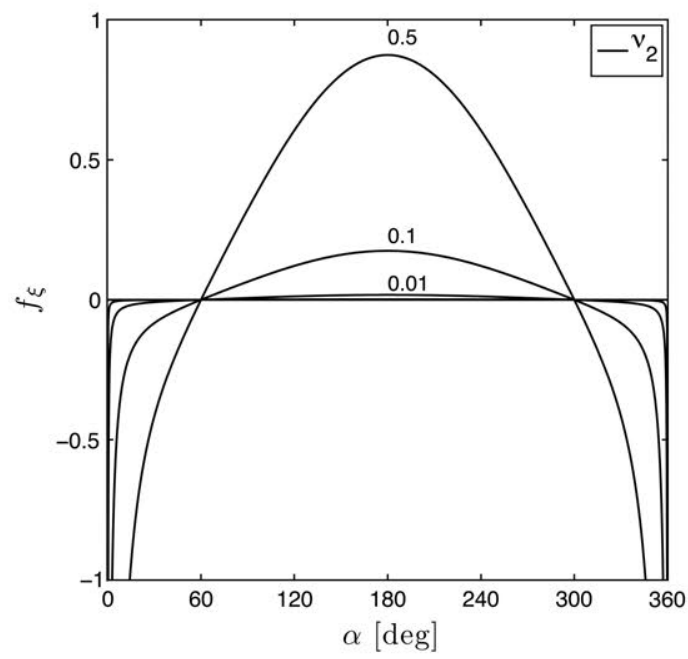

a) $f_{\xi}$

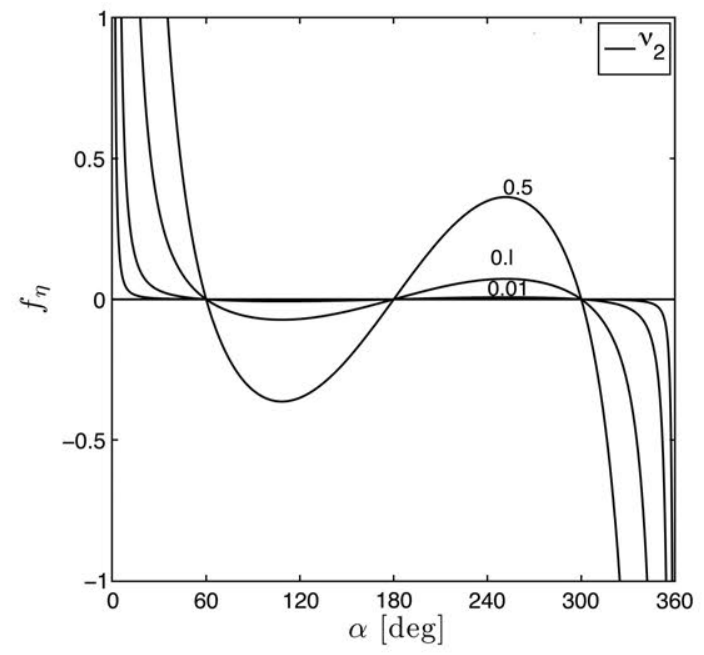

b) $f_{\eta}$

Fig. 4 Graphs of a) nondimensional force component $f_{\xi}$, and b) $f_{\eta}$ required to maintain an equilibrium position with respect to the synodic frame as a funciton of the angle $\alpha$. 


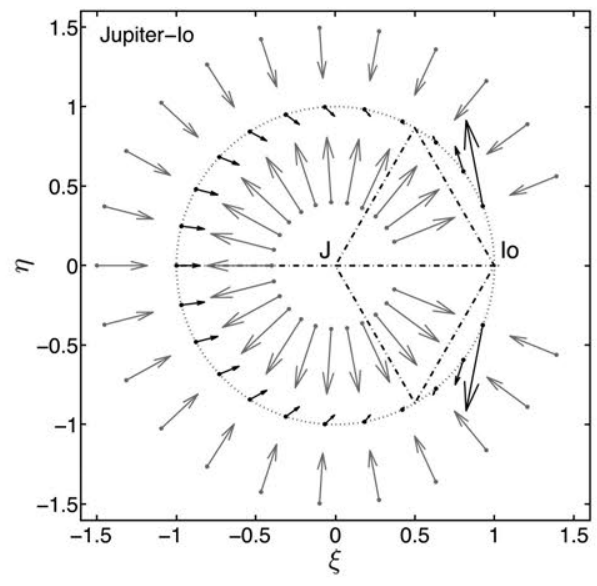

Fig. 5 Nondimensional force components $\left(f_{\xi}, f_{\eta}\right)$ required to stay in equilibrium in the synodic frame at locations (marked by dots) different than classical Lagrangian points.

with a dotted circle. Each arrow in the figure shows the direction and the magnitude (with different scales as explained later) of electrodynamic force required to keep the spacecraft in equilibrium with respect to the synodic frame at locations different from Lagrangian points. Two main zones can be identified. The first one is a narrow zone encircling the circle centered in Jupiter and joining Io with the triangular points. In this region the force has its smaller magnitude (a scale $\times 2500$ has been used for visualization) and has a nonnull tangential component. The second region is the remaining part of the synodic plane, which is cut into an inner and an outer zone by the orbit of Io. In this region the force is predominantly directed in the radial direction, pointing outward in the inner region $(0<r<1)$ and pointing inward in the outer region $(r>1)$.

\section{Self-Powering at Equilibrium Positions}

When the EDT is placed at an equilibrium position away from a Lagrangian point, a nonzero electrodynamic force acts upon it, and the EDT can supply electrical power to the spacecraft itself. The region of allowed equilibria for technologically feasible tethers lies inside the narrow region encircling the circle centered in $M_{1}$ and joining the triangular points with the second primary. When $M_{2} \ll M_{1}$, as in all practical cases of the solar system, the equilibrium points are all placed very close to the orbital path of the second primary, and their effective location is a function of environmental parameters (i.e., plasmasphere and planetary system), tether size, and plasma collection device.

In a general case, the attitude of the tether affects the magnitude and orientation of the electrodynamic force, and consequently the location of the equilibrium points. Assuming the EDT is selfbalanced [27], the force vector acts on the center of mass of the system, and no net electrodynamic torques affect the orientation of the system. Under this hypothesis the equilibrium locations of the EDT can be obtained by considering only the effect of electrodynamic force, substituting the electrodynamic force [Eq. (8)] in the equilibrium equations of the three body [Eqs. (35-37)]. A nonlinear least-squares algorithm has been used to find the roots of the system formed by Eqs. (8) and (35-37). A family of equilibrium locations has been obtained, stemming from the unperturbed triangular points $L_{4}$ and $L_{5}$. Numerical examples are shown in the following paragraph.

A useful simplified model has been derived to describe the powering conditions, under the following hypotheses: 1) the tether system is contained in the synodic plane $(\xi, \eta)$ and points toward $M_{1}$; 2 ) the tether system is above the stationary orbit of $\left.M_{1} ; 3\right)$ aligned and centered magnetic field generated by the first primary and rotating with the angular velocity of the primary $\Omega_{1}$; and 4) orientation of $\mathbf{B}$ is from north to south (as in Jupiter and Saturn), thus, in the synodic plane the unit vector of $\mathbf{B}$ is $\hat{\boldsymbol{b}}=(0,0,-1)$.
Under these simplifications, when the tether is at equilibrium (meaning that it has a zero velocity with respect the synodic frame), the projection of the motional electric field on the tether is simply given by:

$$
E_{t}=B \cdot d \cdot\left(\Omega_{1}-\Omega+\Omega v_{2} \cos \alpha\right)
$$

The Lorentz force vector, with its components expressed in the synodic frame, has the following simple expression, which represents a counterclockwise force, tangential to the orbit of the second primary mass:

$$
{ }^{\mathrm{syn}} \mathbf{F}_{\mathrm{el}}=F_{\mathrm{el}} u \hat{\boldsymbol{u}} \times \hat{\boldsymbol{b}}=F_{\mathrm{el}}(-\sin \alpha ; \cos \alpha ; 0)
$$

where the force magnitude is given by:

$$
F_{\mathrm{el}}=\left(\frac{4}{3} \frac{q_{e}}{\pi} \sqrt{2 \frac{q_{e}}{m_{e}}}\right)\left(1-\frac{2}{5} \zeta\right) \zeta^{3 / 2}\left(w L^{5 / 2}\right)\left(n_{e} B \sqrt{E_{t}}\right)
$$

In these expressions the tether line $\hat{\boldsymbol{u}}=(\cos \alpha ; \sin \alpha ; 0)$ takes into account the direction of the current, which is imposed by the motional electric field pointing outward.

As shown previously a nonzero electrodynamic force is required to keep the EDT system in an off-Lagrangian equilibrium position. The scalar product of this force with the velocity relative to the plasma gives the ideal electrical power generated.

Under the hypotheses of the simplified model the ideal power for a tether placed at an angle $\alpha_{0}$ along the orbit of the second primary is:

$$
P_{\text {id }}=I_{\text {avg }} L B d\left(\Omega-\Omega_{1}-\Omega \cos \alpha_{0} \nu_{2}\right)
$$

In the two-body case, the mass parameter $v_{2}$ vanishes and Eq. (52) simplifies to:

$$
P_{\text {id }}=I_{\text {avg }} L B d\left(\Omega-\Omega_{1}\right)
$$

From the comparison of simplified expressions Eqs. (52) and (53), some important differences between the classical two-body case and the three-Body case can be inferred. In the two-body case the ideal power is simply driven by the difference $\left(\Omega-\Omega_{1}\right)$ between the orbital and plasma angular velocities. In the three-body case the ideal power is reduced by the small term $\cos \alpha_{0} v_{2}$. The reduction term is of geometrical nature, and is due to the displacement between the centers of rotations of the plasmasphere and the corotating frame. The reduction term reaches the maximum value of 0.5 only in the limiting case when the planetary system has $M_{1}=M_{2}$ and the equilibrium point reaches $\alpha_{0} \approx 0$ at the location of the second primary. In all cases of practical relevance within the solar system, the mass parameter is always small, $v_{2}<10^{-3}$ (except for system of asteroids), and the reduction of power due to the three-body effect is negligible. As a result, the power obtained in the three-body case is of the same order of magnitude of the two-body case, but has the additional feature of keeping the spacecraft at an equilibrium point without deorbiting.

\section{EDT in the Jupiter Plasma Torus}

By using the model described in the previous sections, the dynamics of an EDT satellite inside the Jupiter plasma torus has been studied as a special case. Jupiter offers several attractive advantages for EDTs, due to its high rotation rate, strong magnetic field, and nonnegligible plasma density. The presence of the plasma torus all around the Io's orbit makes this region an attractive location for an EDT, both for power generation and propellantless maneuvers. High power levels (kilowatts and more) can be obtained by means of an EDT in the Io torus. These power levels could be of great interest for Jovian missions, which are always handcuffed by the scarcity of power.

The Jupiter magnetic field rotates at the same angular velocity of the planet, with velocity $v_{B}=\Omega_{1} r$, where $\Omega_{1} \approx 1.76 \times 10^{-4} \mathrm{rad} / \mathrm{s}$ 
is the Jupiter angular rotation rate and $r$ is the distance in the equatorial plane. Because of the high rotation rate of the planet, the stationary quote is relatively low at $2.238 R_{j}$, and the velocity of the mangetic field at Io's orbit $\left(5.9 R_{j}\right)$ is about $74 \mathrm{~km} / \mathrm{s}$. As a consequence, a spacecraft co-orbiting on the same orbital path of Io $\left(v_{\mathrm{sc}} \approx 17.3 \mathrm{~km} / \mathrm{s}\right.$ ) has a velocity relative to the Jupiter's magnetic field $v_{\text {rel }} \approx 57 \mathrm{~km} / \mathrm{s}$, that results in a motional electric field $E \approx$ $0.1 \mathrm{~V} / \mathrm{m}$ for a local magnetic field $B \approx 2 \times 10^{-6} \mathrm{~T}$. The resulting electrodynamic force for a spacecraft placed in that location is a thrust force directed along the orbital velocity of the spacecraft. field:

An aligned dipolar model has been used for the Jupiter magnetic

$$
\mathbf{B}(\mathbf{r})=\frac{m}{r^{3}}[3(\hat{\boldsymbol{m}} \cdot \hat{\boldsymbol{r}}) \hat{\boldsymbol{r}}-\hat{\boldsymbol{m}}]
$$

where $\mathbf{m}=\mu_{m} R_{j}^{3} \hat{\boldsymbol{m}}$ is the magnetic dipole moment vector of the planet, $\hat{\boldsymbol{m}}$ is its unit vector, $\mu_{m}$ is the intensity of the dipole [Tesla], $R_{j}$ is the planet equatorial radius, and $\hat{\boldsymbol{r}}$ is the position vector of the spacecraft.

The electron density $N_{e}$ in the torus is derived from the Divine and Garrett model [28], shown in Fig. 6 as a function of the radial distance from Jupiter. The locations of Io and of the four inner moonlets are marked by vertical lines in the figure. The presence of the plasma torus can be inferred from the electron density increase around the Io orbit at $5.9 R_{j}$.

The expressions of equilibrium locations given by the system of Eqs (35-37) plus Eqs. (50) and (51) have been used to size tethers capable of maintaining the spacecraft in equilibrium in the corotating frame of the Jupiter-Io system. The angle $\alpha_{0}$ identifys the equilibrium position of the tether on the circle centered in Jupiter and joining Io with the triangular points. It has been numerically determined for a set of cases as a function of the parameters $L, w, m$, that are the tether length, tether width, and spacecraft mass, respectively. As expected, we found that the EDT parameters influence the magnitude of the force and, consequently, the location of the equilibrium point. Figure 7 shows for a $5 \mathrm{~cm}$ width tape tether the equilibrium angle $\alpha_{0}$ vs the spacecraft mass ranging from 200 to $1200 \mathrm{~kg}$, for five tether lengths $L=8,10,20,30,40 \mathrm{~km}$. As can be seen from Fig. 7, a short tether is sufficient to stay in equilibrium near a triangular point. The more the position moves away from a triangular point toward the second primary (Io), the longer is the tether required or the smaller the satellite mass. This in turn implies that a greater electrodynamic force must be exerted to stay near the second primary body. For example, a tether with a constant length of $10 \mathrm{~km}$, and a suitable spacecraft mass can have equilibrium positions within the range $\alpha=34$ to $52^{\circ}$. Using a controlled variable-length tether and a constant-mass spacecraft, the equilibrium positions can be moved in region very close to the Io's orbital path and inside the plasma torus.

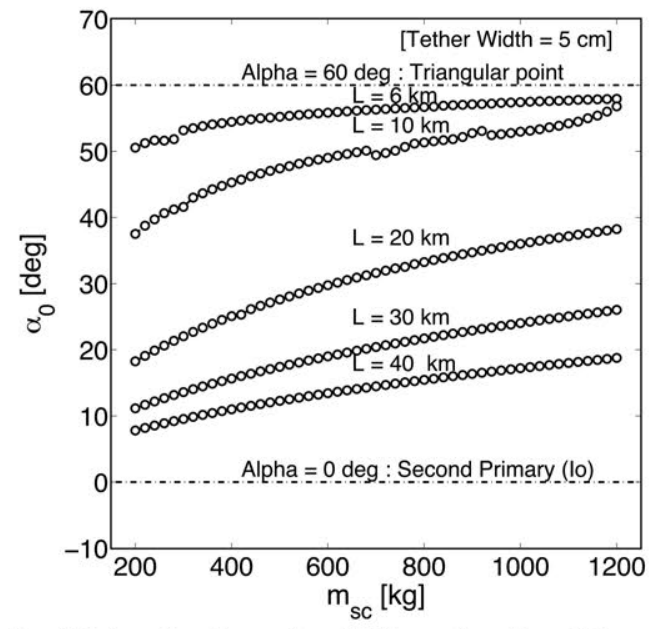

Fig. 7 Equilibrium locations of an EDT as a function of the angle $\alpha$ in the Jupiter-Io system, on the orbital path of the second primary (Io).

The equilibrium positions coincide with the classical triangular points at $\alpha= \pm 60$ deg only when the electrodynamic force is equal to zero.

The useful power extracted by the EDT at equilibrium is shown in Fig. 8, for the librating tether case. The ideal power given by Eq. (12) can be converted into about $30 \%$ for a librating tether, and $20 \%$ for a rotating tether (see [1] for details). The power is proportional to the tether length, with a law $P \propto L^{5 / 2}$, as mentioned earlier and also shown in this figure. Kilowatt level of useful power can be delivered by the EDT to the spacecraft, by extracting the energy from the Jupiter plasmasphere.

\section{Numerical Simulations}

A set of numerical simulations with an upgraded 3D model was carried out. This model integrates the $3 \mathrm{D}$ orbital dynamics of a rigid dumbbell satellite in the three-body system [as described by Eq. (25)], plus the two angular degrees of freedom of its attitude dynamics. The two attitude angles evolve under the effect of the external gravitational torques of the two primaries. The numerical integration of the governing equations is carried out with a standard Adam-Bashforth-Moulton 11th-order predice-evaluate derivatives correct-evaluate derivatives routine [29]. A detailed description of the code implementing this model, together to its validation for a set of relevant cases of both orbital and attitude motion, can be found in [22]. A tilted dipolar magnetic field of Jupiter is considered to model the perturbing effects due to the oscillating field. The Divine and

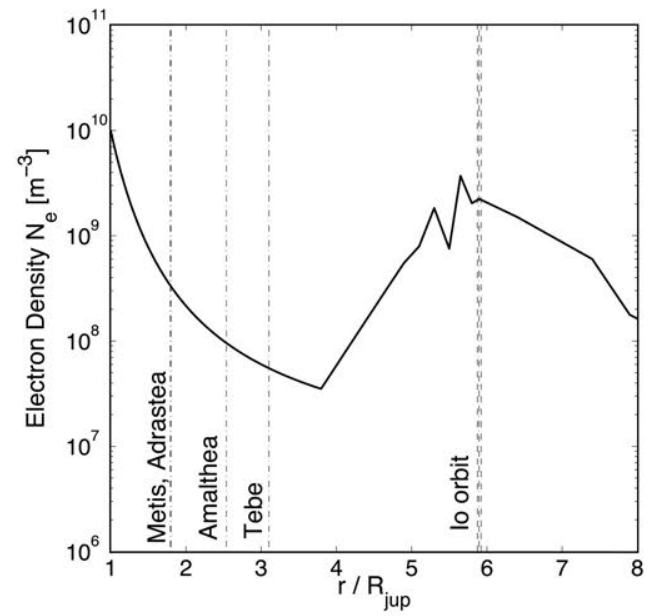

Fig. 6 Electron density at Jupiter according to Divine and Garrett model (adapted from [28]).

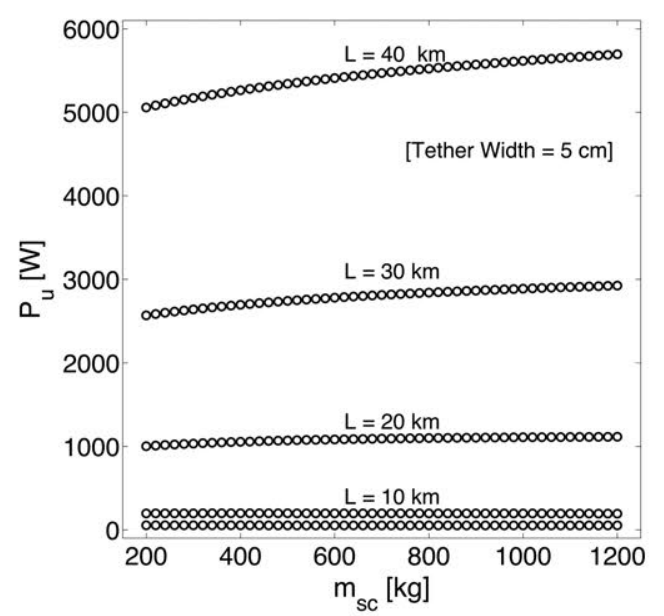

Fig. 8 Useful power at equilibrium for a librating tether vs spacecraft mass. 


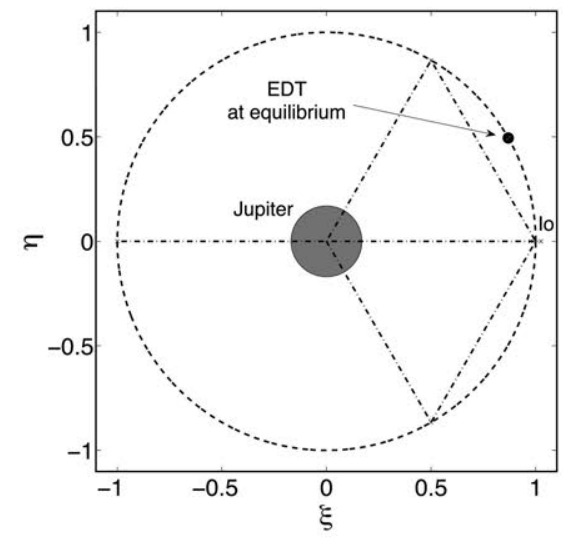

a)

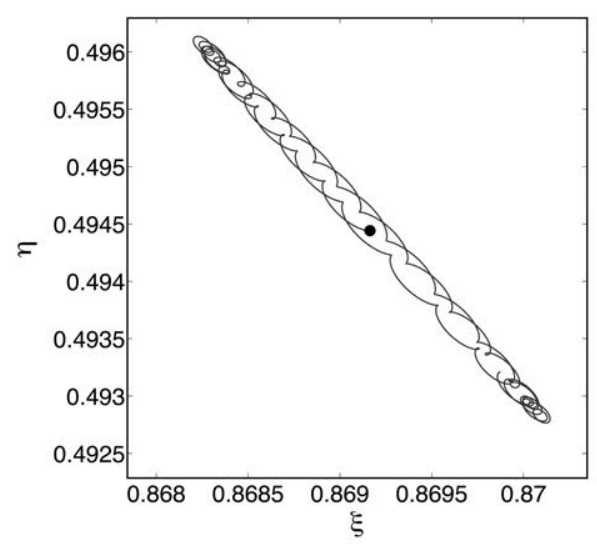

b)

Fig. 9 Graphs of a) EDT at equilibrium in the Jupiter-Io system, and b) small oscillations around the equilibrium position.

Garrett model [28] is adopted for the plasma electron density. The higher fidelity model confirmed the results obtained with the simplified model, finding that the EDT can remain in equilibrium at off-Lagrangian locations. Several values of mass, length, and width were considered, in order to identify the equilibrium position for each configuration.

Figure 9a shows a $20 \mathrm{~km}$ tether of $5 \mathrm{~cm}$ tape width and a $600 \mathrm{~kg}$ mass spacecraft in equilibrium in the corotating frame of Jupiter and Io, for a simulation time $t=40 T_{\text {rev }} \approx 70.76$ days (Io's $T_{\text {rev }}=1.77$ days). The position of the spacecraft is at $\alpha_{0}=29.63^{\circ}$, marked with the arrow-tail sign in the figure. The simplified model predicts an equilibrium position at $\alpha_{0}=29.75^{\circ}$, in accordance with the higher fidelity model. Jupiter and Io are to scale, and their triangular points are also marked in the figure. Figure $9 \mathrm{~b}$ depicts small oscillations around the equilibrium point that exhibits a motion characterized by two eigenfrequencies, analogous to the unperturbed motion around the natural Lagrangian points. The two eigenfrequencies of the classical circular restricted three-body problem [26] are expressed by Eq. (34).

A Lorentz force of about $0.06 \mathrm{~N}$ is exerted on the system, producing a useful power of about $1130 \mathrm{~W}$ for onboard use (see Fig. 10). The perturbation due to the other Galilean satellites is always smaller than this electrodynamic force. The main perturbation is given by the attraction of Europa and Ganymede, being in the worst case 1 order of magnitude less than the electrodynamic force. However, the effect of the other moons could not be completely neglected because of the Laplace resonance 1:2:4 between the orbital periods of Io, Europa, and Ganymede, that can lead to a pumping effect to a satellite placed on the Io orbit. These kind of effects are not addressed in the present study. Numerical simulations with a four-body simulator have revealed that equilibrium can be

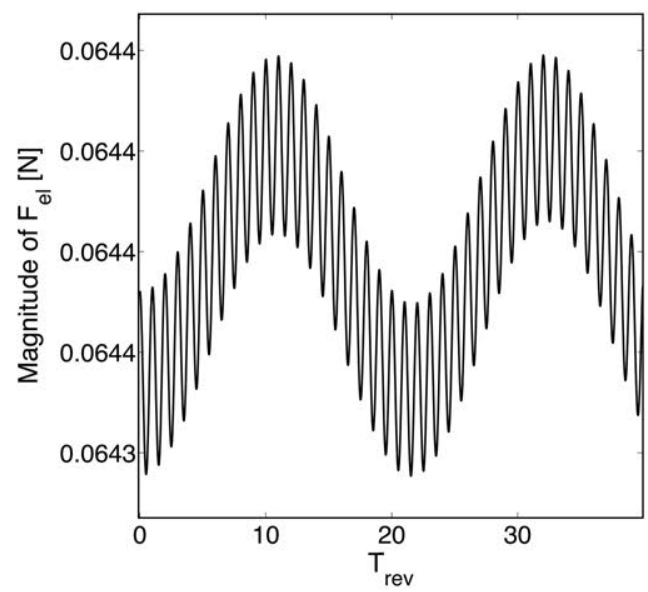

a)

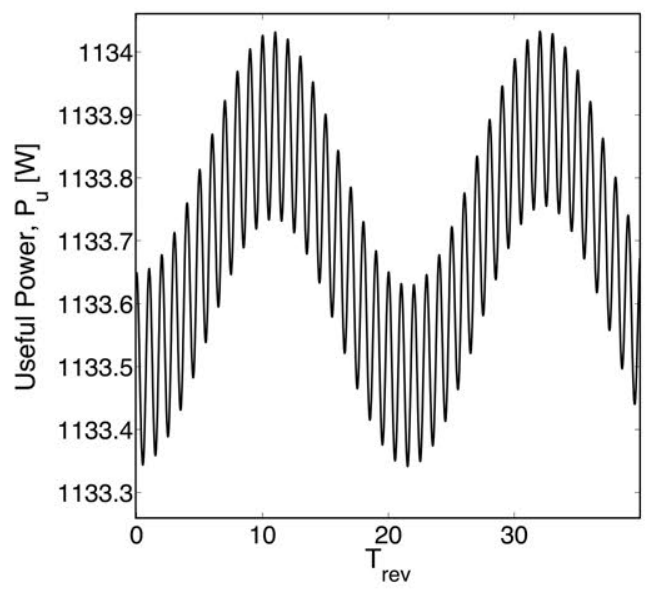

b)

Fig. 10 Graphs of a) electrodynamic force, and b) generated power vs orbital revolutions (Io's $T_{\text {rev }}=1.77$ days) by a $20-\mathrm{km}$ EDT at equilibrium at Jupiter obtained with the higher fidelity model EDT3BODY. These results are in accordance with the simplified model, which gives $F_{\mathrm{el}}=0.064 \mathrm{~N}$ and $P_{u}=1082 \mathrm{~W}$.

reached also in the perturbed case and the equilibrium is disrupted after a long period of time.

\section{Conclusions}

The Lorentz force produced by an electrodynamic tether placed inside the plasmasphere of a three-body planetary system has been considered, with a focus on system dynamics and power generation at equilibrium points. The Lorentz force produced by the tether through the interaction with the superrotating plasma sphere perturbs the natural Lagrangian equilibrium points, and new equilibrium positions appears with respect to the synodic frame corotating with the primary bodies. The equilibrium is among the two gravitational forces of the two primaries, the inertial forces and the local Lorentz force.

The results of simplified model developed to derive the equilibria locations in the synodic plane shows that for technologically feasible tethers those points are located along the circle centered in the first primary and joining the triangular points with the second primary. Differently from the two-body case, in the three-body scenario an EDT can be placed at equilibrium position and at the same time generate electrical power, without deorbiting. The electrical power is generated at the expense of the corotating plasma energy.

A higher fidelity numerical model confirmed the presence of equilibrium positions and the possibility to place an electrodynamic 
tether in their neighborhood while extracting power from the environmental plasma.

The present analysis has been applied to the Jupiter-Io system, where the presence of the plasma torus make this region an attractive place for an EDT to operate. An EDT in the plasma torus can generate in a continuous way kilowatts of useful power (with a tether length of order $20 \mathrm{~km}$ and more), that can be used on board the spacecraft.

\section{Acknowledgment}

The present research was supported by the Ariadna research contract 07/4201 of the Advanced Concept Team of the European Space Agency/European Space Research and Technology Centre with Dario Izzo as Technical Monitor of the contract.

\section{References}

Bombardelli, C., Lorenzini, E. C., and Sanmartìn, J. R. "Jupiter Power Generation with Electrodynamic Tethers at Constant Orbital Energy," Journal of Propulsion and Power, Vol. 25, No. 2, March-April 2009, pp. $415-423$.

doi: $10.2514 / 1.38764$

Peláez, J., Lorenzini, E. C., Lopez-Rebollal, O., and Ruiz, M., "A New Kind of Dynamic Instability in Electrodynamic Tethers," Advances in Astronautical Sciences, Vol. 105, 2000, pp. 1367-1386.

Dobrowolny, M., Arnold, D., Colombo, G., and Grossi, M., "Mechanisms of Electrodynamic Interactions with a Tethered Satellite System and the Ionosphere," Reports in Radio and Geoastronomy, Vol. 6, Aug. 1979.

Estes, R. D., Lorenzini, E. C., Sanmartín, J. R., Peláez, J., MartínezSanchez, M., Johnson, C. L., and Vas, I. E., "Bare Tethers for Electrodynamic Spacecraft Propulsion," Journal of Spacecraft and Rockets, Vol. 37, No. 2, March-April 2000, pp. 205-211.

doi: $10.2514 / 2.3567$

Takeichi, N., "Practical Operation Strategy for Deorbit of an Electrodynamic Tethered System," Journal of Spacecraft and Rockets, Vol. 43, No. 6, Nov.-Dec. 2006, pp. 1283-1288.

doi: $10.2514 / 1.19635$

Johnson, L., Estes, R. D., Lorenzini, E. C., Martínez-Sanchez, M., and Sanmartín, J. R., "Propulsive Small Expendable Deployer System Experiment," Journal of Spacecraft and Rockets, Vol. 37, No. 2, 2000, pp. $173-176$.

doi: $10.2514 / 2.3563$

Gilchrist, B. E., Bilen, E., Sven, G., and Johnson, L., "Space Electrodynamic Tether Propulsion Technology: System Considerations and Future Plans," AIAA/ASME/SAE/ASEE Joint Propulsion Conference and Exhibit, 35th, AIAA, Reston, VA, 1999.

Alfven, H., "Spacecraft Propulsion: New Methods," Science, Vol. 176, No. 4031, April 1972, pp. 167-168.

doi:10.1126/science.176.4031.167

Gabriel, S. B., Jones, R. M., and Garrett, H. B., "Alfven Propulsion at Jupiter," International Conference on Space Tethers for Science in the Space Station Era, edited by L. Guerriero, and I. Bekey, Vol. 14, NASA, Oct. 1987.

Sanmartín, J. R., and Lorenzini, E. C., "Exploration of Outer Planets Using Tethers for Power and Propulsion," Joumal of Propulsion and Power, Vol. 21, No. 3, May-June 2005, pp. 573-576.

doi:10.2514/1.10772

Sanmartín, J. R., Charro, M., Bramanti, C., Bombardelli, C., Lorenzini, E. C., and Garrett, H. B., "Electrodynamic Tether Microsats at the Giant Planets," Advanced Concept Team ESA, Tech. Rept. 05/3203, Sept. 2006.
Bombardelli, C., Lorenzini, E. C., Curreli, D., Sanjurjo-Rivo, M., Lucas, F., and Lara, M., "Io Exploration with Electrodynamic Tethers," AIAA/AAS Astrodynamic Specialist Conference, AIAA, Reston, VA, 2008.

Penzo, P., "A Survey of Tether Applications to Planetary Exploration," Tethers in Space; Proceedings of the International Conference, edited by P. Bainum, I. Bekey, L. Guerriero, and P. Penzo, NASA, Sept 1987. Colombo, G., "The Stabilization of an Artificial Satellite at the Inferior Conjunction Point of the Earth-Moon system," Smithsonian Astrophysical Observatory, Special Rept. 80, Sept. 1961.

Farquhar, R. W., "The Control and Use of Libration-Point Satellites," NASA TR R-346, Sept. 1970.

Misra, A. K., Bellerose, J.,Modi, V. J.,"Dynamics of a Tethered System Near the Earth-Moon Lagrangian Points," Advances in Astronautical Sciences, Vol. 109, AIAA, Reston, VA, 2001, pp. 415-435.

Wong, B., and Misra, A. K., "Dynamics of a Multi-Tethered System Near the Sun-Earth Lagrangian Point," Vol. 13th AAS/AIAA Space Flight Mechanics Meeting, AIAA, Reston, VA, Feb. 2003, pp. 16751694.

Wong, B., and Misra, A. K., "Dynamics of a Libration Point MultiTethered System," Proceedings of 2004 International Astronautical Congress, IAC Paper 04-A.5.09, 2004.

Peláez, J., and Scheeres, D. J., "A Permanent Tethered Observatory at Jupiter. Dynamical Analysis," AAS/AIAA Space Flight Mechanics Meeting, AIAA, Reston, VA, Jan.-Feb. 2007, pp. 1307-1330.

Peláez, J., and Scheeres, D. J., "On the Control of a Permanent Tethered Observatory at Jupiter," AAS/AIAA Astrodynamics Specilist Conference, AIAA, Reston, VA, Aug. 2007, pp. 1835-1858.

Sanjurjo-Rivo, M., Lucas, F., Peláez, J., Bombardelli, C., Lorenzini, E. C., Curreli, D., Sheeres, D. J., and Lara, M., "On the Dynamics of a Tethered System Near the Collinear Libration Point," AIAA/AAS Astrodynamic Specialist Conference, AIAA Paper 2008-7380, 2008. Peláez, J., Sanjurjo-Rivo, M., Lara, M., Lorenzini, E. C., Curreli, D., Sheeres, D. J., Bombardelli, C., and Izzo, D., "Dynamics and Stability of Tethered Satellites at Lagrangian Points," Tech. Rept. 07-4201, Advanced Concept Team ESA, Nov. 2008,

Sanmartín, J. R., Martínez-Sanchez, M., and Ahedo, E., "Bare Wire Anodes for Electrodynamic Tether," Journal of Propulsion and Power, Vol. 9, 1993, pp. 352-320.

doi: $10.2514 / 3.23629$

Mott-Smith, H. M., and Langmuir, I., "The Theory of Collectors in Gaseous Discharges," Physical Review, Vol. 28, No. 4, 1926, pp. 727 763.

doi:10.1103/PhysRev.28.727

Chen, F. F., "Langmuir Probe Analysis for High Density Plasmas ," Physics of Plasmas, Vol. 8, No. 6, June 2001, pp. 3029-3041. doi: $10.1063 / 1.1368874$

Szebehely, V., "Motion near the Equilibrium Points," Theory of Orbits: The Restricted Problem of Three Bodies, Academic Press, New York, 1967, pp. 231-308.

Peláez, J., "Self Balanced Electrodynamic Tethers," ALAA/AAS Astrodynamics Specialist Conference and Exhibit, AIAA Paper 2004 5309, Aug. 2004.

Divine, N., and Garrett, H. B., "Charged Particle Distributions in Jupiter's Magnetosphere," Journal of Geophysical Research, Vol. 88, Sept. 1983, pp. 6889-6903. doi:10.1029/JA088iA09p06889

Shampine, L. F., "Numerical Solution of Ordinary Differential Equations," Chapman and Hall, New York, 1994. 